\title{
Chères lectrices, chers lecteurs, chers membres de la SSG
}

Vous tenez aujourd'hui entre les mains la première édition d'une toute nouvelle revue spécialisée:

\section{GÉRONTOLOGIE Appliquée!}

GÉRONTOLOGIE Appliquée n'est pas seulement une nouvelle revue spécialisée, mais c'est aussi le nouveau magazine des membres de la Société suisse de gérontologie (SSG)!

GÉRONTOLOGIE Appliquée est l'expression visible des efforts de la SSG pour faire avancer la professionnalisation, l'interconnexion et la collaboration multidisciplinaire dans le domaine de la «vieillesse» et pour mettre un support adapté à la disposition de la communauté grandissante des gérontologues. Allant dans le sens de la SSG, GÉRONTOLOGIE Appliquée traite de sujets brûlants gravitant autour de la vieillesse et informe sur les évolutions et les opinions actuelles dans ce domaine afin d'inciter des débats de fond spécialisés.

Cette nouvelle revue spécialisée est conçue pour s'adresser à des experts dans le domaine de la vieillesse, des étudiants mais aussi tous ceux qui ne sont pas explicitement employés dans le domaine de la vieillesse et qui sont cependant confrontés au quotidien à des questions concernant la vieillesse et qui cherchent une nouvelle impulsion pour leur domaine d'activité.

\section{Mais à quoi sert la GÉRONTOLOGIE Appliquée?}

Dans notre société où la durée de vie s'allonge, nous avons besoin plus que jamais de connaissances spécifiques en gérontologie. Face à l'évolution démographique, ce ne sont pas uniquement de nouveaux résultats de recherche qui sont demandés mais également et surtout leur application dans la pratique. En Suisse, il existe actuellement un vide concernant la transmission adaptée des nombreuses informations existantes dans la pratique de la gérontologie appliquée. La nouvelle GÉRONTOLOGIE Appliquée souhaite précisément combler ce vide et proposer un forum largement étayé qui prend en considération de manière ciblée l'application des connaissances gérontologiques et le transfert entre théorie et pratique.

En tant qu'organe membre de la SSG, cette nouvelle revue remplace la précédente Gérontologie Information et est, en tant que telle, incluse dans la cotisation annuelle pour les membres de la SSG. Les non-membres peuvent se procurer la revue par abonnement libre ou en achetant juste l'édition.

La nouvelle revue GÉRONTOLOGIE Appliquée paraîtra à l'avenir quatre fois par an avec un thème principal. Cette première édition est consacrée au thème "Vieillesse et technologie », le thème du congrès SSG de cette année.
GÉRONTOLOGIE Appliquée souhaite dans la mesure du possible diffuser ces informations dans toute la Suisse. Pa conséquent, tous les articles seront publiés dans la langue de l'auteur (allemand, français ou italien). Par ailleurs, pour chaque contribution, un bref résumé sera traduit et paraîtra en allemand ou en français. Ainsi, vous pourrez obtenir un bref aperçu du contenu de l'article et ensuite décider si vous souhaitez lire en ligne la version complète de l'article dans la langue souhaitée. En effet, toutes les contributions seront intégralement mises à disposition en ligne aussi bien en allemand qu'en français. Ce service est compris dans l'abonnement ou lors de l'achat d'une seule édition. La SSG souhaite ainsi contribuer aux échanges dans l'ensemble de la Suisse entre les gérontologues appliqués en activité.

Vous retrouverez également dans cette revue différents référents et leurs articles.

C'est pour moi une très grande joie d'avoir réussi avec la nouvelle formule de GÉRONTOLOGIE Appliquée à réaliser une étape importante pour la gérontologie appliquée en Suisse. A cette occasion, je souhaite remercier de tout cœur aussi bien le conseil d'administration de la SSG pour les importantes discussions au préalable, les éditions Hogrefe Verlag à Berne pour le précieux soutien dans le processus d'élaboration, notre équipe de rédaction pour leur engagement et leur compétence ainsi que tous les auteurs qui ont contribué de manière déterminante à la présentation aujourd'hui de cette première édition de GÉRONTOLOGIE Appliquée !

J'espère de tout cœur que la lecture de la nouvelle revue vous plaira et vous stimulera. J'attends volontiers vos remarques et suggestions pour les prochaines éditions.

Le thème de la deuxième édition 2016 se penchera sur la question du « Profil du gérontologue ».

J'aurais peut-être également le plaisir de recevoir l'un de vos articles en tant qu'auteur pour GÉRONTOLOGIE Appliquée!
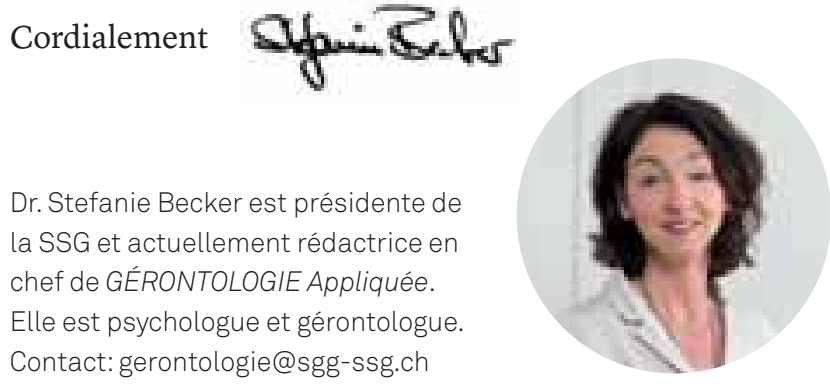